\title{
Caracterização de clorita portadora de Zn por espectroscopia Mössbauer (EM) e espectroscopia infravermelho (IV) - uma ocorrência associada ao depósito de Pb-Zn-Ag de Canoas, PR, Brasil
}

\author{
Rosely Aparecida Liguori Imbernon ${ }^{1}$, Alain Blot ${ }^{2}$, \\ Vitor Paulo Pereira ${ }^{3}$ \& Daniel Ribeiro Franco ${ }^{4}$
}

\begin{abstract}
Resumo Amostras de clorita associadas ao minério sulfetado de $\mathrm{Pb}-\mathrm{Zn}$-Ag do depósito de Canoas 1, Vale do Ribeira, Paraná, Brasil, foram analisadas por espectroscopia Mössbauer (EM) e infravermelho (IV). Os estudos empreendidos possibilitaram discutir questões relacionadas ao processo de cloritização associado à estrutura cristalina, e as formas pelas quais o $\mathrm{Zn}$ foi incorporado no mineral neoformado. Tais questões possibilitam retomar aspectos geoquímico/mineralógicos desse processo natural, onde a cristaloquímica e os mecanismos de formação de cloritas têm sido utilizados em vários campos das ciências aplicadas. Os resultados evidenciaram que se trata da ocorrência de clorita, que ao incorporar Zn na estrutura altera padrões estruturais que se refletiram nas análises por EM e IV. Os dados obtidos por EM evidenciaram que o Fe está localizado exclusivamente nos sítios octaédricos em arranjo trans, e a entrada de $\mathrm{Zn}$ durante o processo de cloritização ocorre, também, na camada brucítica.
\end{abstract}

Palavras-chave: clorita zincífera, espectroscopia infravermelho, espectroscopia Mössbauer, depósito de Canoas-Brasil.

\begin{abstract}
Characterization of Zn-bearing chlorite by Mössbauer (ME) and infrared spectroscopy (IR) occurrence associated to the Pb-Zn-Ag deposits of Canoas of, PR, Brasil. In order to provide new insights on mineralogical aspects of geochemical mapping/natural processes related to the chlorite formation (e.g. crystallochemistry and mechanisms of formation of these materials, which has been applied in different studies of environmental profiles), we investigated chlorite samples associated to the $\mathrm{Pb}-\mathrm{Zn}-\mathrm{Ag}$ sulfide ore from Canoas 1 deposit (Vale do Ribeira, state of Paraná, Brazil). By means of Mössbauer (MS) and infrared (IV) spectroscopy, we addressed some issues as those related to the chloritization processes, as well as how $\mathrm{Zn}$ would be incorporated into its crystalline structure. Results carried out by ME and IV spectroscopy clearly pointed out for a chlorite occurrence, which in fact incorporates $\mathrm{Zn}$ into its structure and also alters the structural patterns for this mineral. Moreover, ME data sets indicated the presence of Fe which is located only in octahedral sites, in trans-configuration, and the $\mathrm{Zn}$ emplacement by the chloritization process also occurs in the brucite layer.
\end{abstract}

Keywords: zinciferous chlorites, infrared spectroscopy, Mössbauer spectroscopy, Canoas deposit-Brazil.

INTRODUÇÃO Ao estudarmos a cloritização de flogopita com incorporação de zinco associada ao minério sulfetado de $\mathrm{Pb}-\mathrm{Zn}$-Ag no depósito de Canoas, Vale do Ribeira, Paraná, Brasil, nos deparamos com a possibilidade de uma nova ocorrência mineral (Blot et al. 1995, Imbernon et al. 1999). As amostras estudadas apresentaram características similares àquelas descritas por Dunn et al. (1987) para a franklinfurnaceíta, de Franklin, New Jersey, USA, e para a baileychlore proveniente de Chillagoe, Austrália e estudada por Rule \& Radke (1988).

Os estudos empreendidos, utilizando dados químicos, difratometria de raios $\mathrm{X}$, microscopia eletrônica de varredura e análises térmicas, tiveram por objetivo compreender o processo de formação de clorita de Canoas, a partir de flogopita. Além disso, se estudou a forma como ocorreu o aporte de zinco na estrutura cristalina do mineral neoformado, assim como em que sítios de ocupação este metal foi incorporado (Blot \& Imbernon 2000).

A elevada variabilidade química e estrutural do mineral clorita é, em muitos casos, um obstáculo no sentido de compreendermos seus mecanismos de formação, transformação e alteração.

Considerando a estrutura genérica dos filossilicatos, que compreende diferentes arranjos de camadas constituídas por cátions em coordenação octaédrica e tetraédrica, a clorita representa um grupo de minerais que ocorre em uma variedade bastante grande de sítios geológicos, com propriedades químicas, físicas e cristalográficas similares.

1 - Escola de Artes, Ciências e Humanidades - EACH, Universidade de São Paulo, São Paulo (SP), Brasil. E-mail: imbernon@usp.br

2 - Institut de Recherche pour le Développement - IRD, Paris, França. E-mail: blotalain@free.fr

3 - Instituto de Geociências, Universidade Federal do Rio Grande do Sul - UFRGS, Porto Alegre (RS), Brasil. E-mail: vitor.pereira@ufrgs.br

4 - Coordenação de Geofísica - COGE, Observatório Nacional, Rio de Janeiro (RJ), Brasil. E-mail: drfranco@on.br 
Existem diferentes propostas de fórmula geral do grupo das cloritas. Alguns autores (Brindley \& Brown 1984, Newman \& Brown 1987) propõem como fórmula estrutural:

$$
\left(R_{6, y, \ldots}^{2+} R_{y}^{3+} \square_{z}\right)_{2}\left(\mathrm{Si}_{4, \ldots k} R_{k}^{3+}\right)_{2} \mathrm{O}_{20}(\mathrm{OH})_{16}
$$

onde os parâmetros $y$ e $k$ indicam o grau de substituição dos cátions trivalentes nas camadas octaédricas e tetraédricas respectivamente, e $z$ o número de vacâncias ( $\square$ ) (Foster 1962). Em geral, a distribuição dos cátions divalentes $\left(R^{2+}\right)$ e trivalentes $\left(R^{3+}\right)$ não são conhecidas de fato, tanto no interior de cada camada quanto entre elas. Contudo, podemos decompor a fórmula apresentada a partir da contribuição dos dois tipos de camadas que se alternam na estrutura da clorita. A composição para a camada brucítica é dada pela fórmula:

$$
\left(R_{6, \ldots, y^{\prime}, 2^{\prime}}^{2+} R_{y^{\prime}}^{3+} \square_{z^{\prime}}\right)(\mathrm{OH})_{12}
$$

enquanto a composição genérica para a camada 2:1 é:

$$
\left(R_{6, \ldots y^{*} \ldots z^{\prime \prime}}^{2+} R_{z^{\prime \prime}}^{3+} \square_{y^{\prime \prime}}\right)\left(\mathrm{Si}_{4, k} R_{k}^{3 \dagger}\right)_{2} \mathrm{O}_{20}(\mathrm{OH})_{4}
$$

e os coeficientes combinados segundo $y^{\prime}+y^{\prime \prime}=y$ e $z$ ' $+z^{\prime \prime}=z$ (Zazzi et al. 2006).

Nos tetraedros normalmente ocorre o $\mathrm{Si}^{4+}$, que pode ser substituído por um cátion $R^{3+}$, usualmente, o $\mathrm{Al}^{3+}$ ou, em menor quantidade, outros cátions. As substituições nos octaedros podem ser tanto de íons divalentes quanto de íons trivalentes. De forma geral, sítio octaédrico pode conter três cátions com valência $2+$, portanto, a existência de íons com valência $3+$ ou $4+$ implica na presença de vacâncias.

Cristais de clorita trioctaédricas (três cátions com valência $2+$ no octaedro) são os mais comumente encontrados e incluem clinocloro, chamosita e penninita (Bailey 1988), que diferem entre si pelo íon que ocupa o sítio octaédrica: $\mathrm{o} \mathrm{Mg}^{2+}$ no clinocloro, o $\mathrm{Fe}^{2+}$ na chamosita e o $\mathrm{Mn}^{2+}$ na penninita.

A compensação das cargas negativas das camadas de talco (tetraedro-octaedro-tetraedro), decorrentes das substituições de elementos nestes sítios, se dá pelas cargas positivas da camada brucítica. No entanto, é difícil separar a contribuição de uma ou de outra camada para o balanço de cargas (Bailey 1988).

Os novos dados obtidos neste estudo, para cristais de clorita no depósito de Canoas, foram analisados visando o entendimento do comportamento cristaloquímico do $\mathrm{Zn}$ no processo de cloritização de flogopita desta localidade. A compreensão do processo de inserção do $\mathrm{Zn}$ na estrutura cristalina da clorita pode ser utilizada como critério para a prospecção deste elemento. Da mesma forma, poderá auxiliar a remediação e descontaminação de áreas degradadas por metais.

Aspectos da geologia e da mineralização na área de ocorrência das cloritas A região do Vale do Ribeira é conhecida desde o início do século XX como Província Metalogenética e área de produção de $\mathrm{Pb}, \mathrm{Zn}$ e $\mathrm{Ag}$ (Fig. 1). O minério foi explorado em diversas minas na região: Furnas e Lageado, no estado de São Paulo, Panelas, Rocha, Paqueiro, Perau e Canoas, no estado do Paraná.

O depósito está localizado em áreas próximas às margens do rio Ribeira, $25 \mathrm{~km}$ a sudeste do município de Adrianópolis, Paraná, Brasil, divisa com o estado de São Paulo (Fig. 1).

$\mathrm{Na}$ área do depósito de Canoas, ocorrem três corpos mineralizados, Canoas 1, 2 e 3 (Fig. 2), sendo que os dois primeiros depósitos foram explorados até meados de 1995 (Bettencourt et al. 1992).

As mineralizações em $\mathrm{Pb}$ na região foram classificadas em dois tipos principais: os depósitos tipo Panelas e os depósitos tipo Perau (Fleischer 1976). Os depósitos tipo Panelas são constituídos por mineralizações epigenéticas, representadas por corpos mineralizados, em geral, discordantes e com forte controle estrutural e litológico, que se encaixam em rochas metacarbonáticas do Grupo Açungui. Os depósitos tipo Perau são caracterizados por mineralizações singenéticas, concordantes com as rochas encaixantes, associadas às formações ferríferas e/ou corpos de barita que se dispõem ao longo de um horizonte carbonático próximo à porção basal da Formação Perau (meso-Proterozoico).

O minério consiste, principalmente, na disseminação de sulfetos em rochas com diferentes composições, caracterizando três tipos: baritífero, silicático e cálcio-silicático. As mineralizações, quando associadas às rochas cálcio-silicáticas, são predominantemente bandadas com sulfetos dispondo-se, preferencialmente, em determinadas camadas paralelas ao plano de foliação ou em zona interboudin; quando associadas às rochas baritíferas, apresentam estrutura bandada com alternância de camadas ricas e pobres em sulfetos, ou estrutura brechada, geralmente com fragmentos de metachert. Em menor escala, o minério pode também ocorrer associado a rochas metapelíticas, com sulfetos alojados ao longo da foliação, em sua maioria com feições de cisalhamento rúptil e cimentação sulfetada (Daitx 1996).

Os principais minerais de minério são a pirita, a galena e a esfalerita; subordinadamente ocorrem calcopirita, pirrotita, marcassita, titanita e rutilo. A Ag foi identificada em fases minerais na forma de tetraedrita/freibergita, pirargita, stephanita, polibasita, argentita (Daitx 1996). Embora os sulfetos ocorram em todos os tipos de minério, Daitx (1996) observou a associação preferencial entre algumas fases minerais e determinados tipos de minério. Assim, a galena é o sulfeto com maior predominância nos minérios baritíferos bandados, associado à esfalerita e com pirita em teores variáveis. Nos minérios baritíferos brechados ocorre o predomínio da esfalerita sobre a galena; a pirita é mais frequente e a calcopirita ocorre como acessório comum. Nos minérios silicáticos predomina a esfalerita e sulfetos de ferro, como a pirita e a 


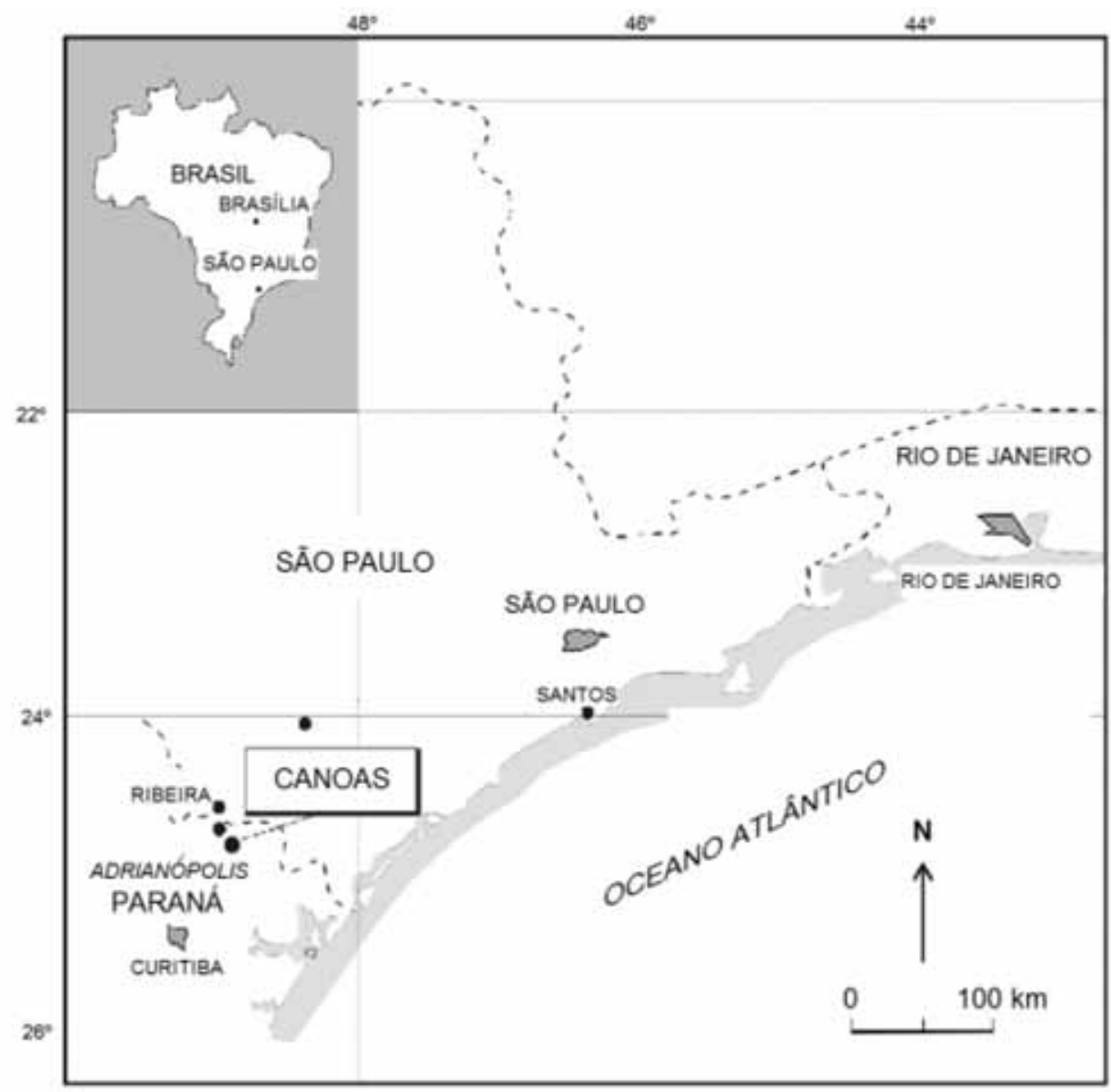

Figura 1 - Mapa de localização do depósito de Pb-Zn-Ag de Canoas 1 - PR (adaptado de Imbernon 1998).

pirrotita, sobre a galena, sendo a calcopirita o acessório mais comum.

A composição mineralógica do minério primário apresenta reflexo nos materiais que evoluíram a partir dos processos intempéricos. Dos três corpos mineralizados, somente em Canoas 1 o material de capeamento apresenta transformações típicas de alteração de sulfetos. Por estar topograficamente sobre o minério no interior da mina, e apresentar uma mineralogia com sulfatos secundários (jarosita, piromorfita), esse material foi descrito e caracterizado como um chapéu de ferro por Imbernon et al. (1999).

MATERIAIS E MÉTODOS Nos trabalhos de campo foram realizadas amostragens de clorita no interior da mina de Canoas 1 e não foram observadas outras ocorrências nos demais depósitos de Canoas 2 e 3 . Os minerais ocorrem em níveis de clorita mais ou menos contínuos, com espessura máxima de $1 \mathrm{~cm}$, distribuídos entre os minerais da ganga, ou na forma de agregados. A ocorrência destes níveis, na entrada da mina de Canoas 1 está associada a material de capeamento superficial bastante intemperizado. No interior da mina e nas amostragens em furos de sondagem não se identificou ocorrência semelhante.

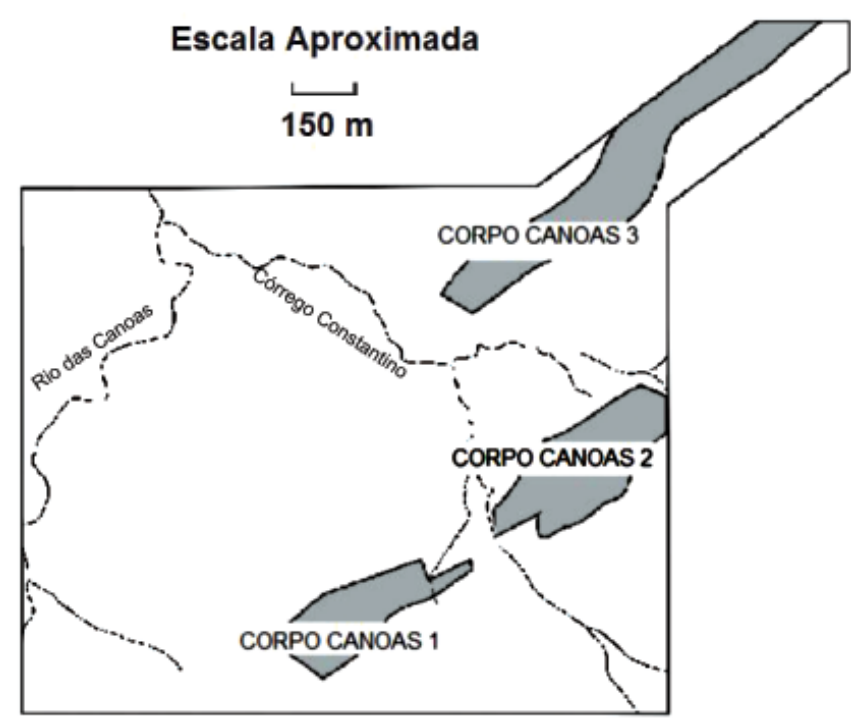

Figura 2 - Esquema de distribuição espacial dos corpos de minério de Canoas 1, 2 e 3. Compilado a partir do mapa de Daitx (1996) e informações fornecidas pelo geólogo da Plumbum S/A, Normando Queiroga. 
O material bruto foi impregnado com resina para confecção de lâminas delgadas para observação em microscópio óptico, sendo selecionados os locais para análises químicas pontuais por microscopia eletrônica de varredura (MEV). As amostras foram desagregadas para separação de concentrados de clorita. $\mathrm{O}$ concentrado de minerais foi utilizado para análises por espectroscopia Mössbauer (EM) e espectroscopia no infravermelho (IV).

A composição química das cloritas foi determinada sobre lâminas delgadas, de forma que pudéssemos obter uma precisa relação entre o processo de evolução da cloritização e os teores de zinco na clorita. Para isso, foi utilizado um equipamento Stereocan 200, equipado com EDS AN 1000, marca Cambridge, sem utilização de padrões, corrente estável, programa ZAF acoplado. Este equipamento pertence ao laboratório do Institut de Recherche pour le Développement - IRD, Bondy, França.

As medidas obtidas por EM - técnica que vem sendo empregada de maneira crescente na identificação de fases portadoras de $\mathrm{Fe}$ na caracterização de materiais formados em ambientes naturais como solos, sedimentos e rochas (Franco et al. 2007) - foram realizadas em equipamento WISSEL, operando com aceleração constante e usado em geometria de transmissão com fonte ${ }^{57} \mathrm{Co} / \mathrm{Rh}$ (intensidade inicial em torno de $50 \mathrm{mCi}$ ) do Instituto de Física da Universidade de São Paulo - USP. Os espectros foram obtidos em temperatura ambiente e em baixa temperatura $(77 \mathrm{~K})$ em criostato de nitrogênio líquido. $\mathrm{O}$ deslocamento isomérico $(\delta)$ foi calibrado em relação à escala de velocidade, utilizando $\alpha$-Fe à temperatura ambiente. Os ajustes dos espectros Mössbauer foram realizados segundo modelagem de sítios cristalográficos, através de ajustes de linhas Lorentzianas.

As análises por espectroscopia no infravermelho, por transformada de Fourier (IV), foram realizadas nos laboratório do IRD - Bondy, França, em equipamento Diamond Perkin Elmer Spectrum 2000 FT-IR. Os materiais-referência utilizados foram a caulinita (Aldrich) e a illita (fornecidos por ENSCI - Limoges, France). As análises por IV foram efetuadas em um conjunto de 8 amostras de clorita, mais ou menos puras, com a presença de carbonatos, fosfatos, sulfatos e outros silicatos. Optou-se pela não purificação das amostras e, devido à complexidade da população analisada, e de maneira a aumentar a eficiência na análise dos dados de maior dificuldade de leitura, efetuamos um tratamento sobre os valores estatísticos das medidas e suas flutuações a partir dos coeficientes de variação calculados.
RESULTADOS As observações ao microscópio óptico permitiram verificar que os cristais de clorita se desenvolveram pseudomorficamente sobre a flogopita, indicando uma gênese a partir da lixiviação de alguns cátions. Este processo se caracteriza pela progressiva e simultânea alteração da flogopita e pela dissolução do sulfeto primário portador de $\mathrm{Zn}$, a esfalerita primária (Blot \& Imbernon 2000). Com isto, ocorre a incorporação de zinco $(\mathrm{Zn})$ pela clorita neoformada a partir da flogopita, com a eliminação quase total de potássio da mesma. Isso permitiu-nos concordar com Blot et al. (1995) e considerar que a clorita zincífera de Canoas é produto da alteração intempérica de um sulfeto e de um filossilicato (Figs. 3 e 4).

Os dados obtidos ao MEV/EDS para a composição química das cloritas estão sintetizados na tabela 1 na forma de médias simples e respectivos desvios a partir do conjunto de análises apontado por Imbernon (1998). Consideramos para as interpretações o conjunto de análises com fechamento (totais) entre 87 e $90 \%$, e uma perda ao fogo total em torno de 10\% (Blot \& Imbernon 2000).

$\mathrm{O}$ espectro Mössbauer obtido para a clorita à temperatura ambiente não se revelou como diagnóstico do comportamento do conteúdo da clorita; enquanto o espectro obtido à temperatura de $77 \mathrm{~K}$ indicou a ocorrência desta fase mineral através dos parâmetros relativos aos três dubletos quadrupolares (Fig. 5). Os dados obtidos, efetuados os cálculos e ajustes para os dubletos, estão sumarizados na tabela 2.

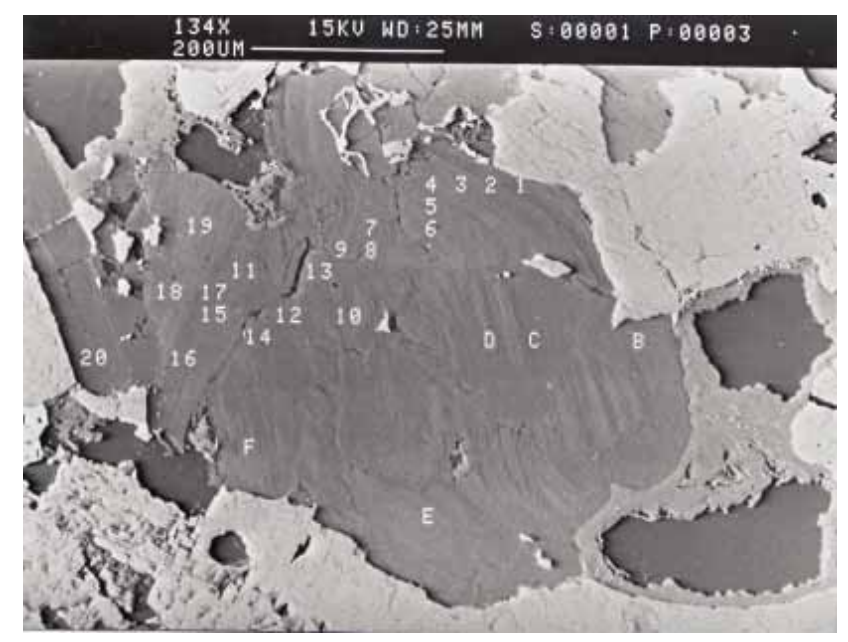

Figura 3 - Localização dos pontos analisados por microscopia eletrônica de varredura (MEV/ZAF) em flogopita parcialmente cloritizada (P2 - P13, P18, P19, $A, B, D$, e F= clorita; P14 - P17 e C=flogopita).

Tabela 1 - Média dos dados químicos para a clorita de Canoas 1 a partir de análises ao MEV/EDS (n=31).

\begin{tabular}{lccccccccc}
\hline Óxidos & $\mathrm{SiO}_{2}$ & $\mathrm{Al}_{2} \mathrm{O}_{3}$ & $\mathrm{Fe}_{2} \mathrm{O}_{3}$ & $\mathrm{~K}_{2} \mathrm{O}$ & $\mathrm{MgO}$ & $\mathrm{TiO}_{2}$ & $\mathrm{ZnO}$ & $\mathrm{CaO}$ \\
\hline$\%$ & 25,90 & 11,44 & 16,93 & 0,533 & 16,23 & 0,61 & 17,12 & 0,06 \\
\hline$\sigma$ & 2,471 & 2,562 & 6,347 & 1,14 & 2,065 & 0,292 & 4,208 & 0,116 \\
\hline
\end{tabular}


Tabela 2 - Parâmetros obtidos a partir do espectro Mössbauer à 77 K para a clorita de Canoas 1 .

\begin{tabular}{lccc}
\hline & $\mathrm{D}_{1}$ & $\mathrm{D}_{2}$ & $\mathrm{D}_{3}$ \\
\hline $\mathrm{DQ}(\mathrm{mm} / \mathrm{s})$ & $2,664(1)$ & $0,840(1)$ & $0,458(1)$ \\
\hline$\delta(\mathrm{mm} / \mathrm{s})$ & $1,107(1)$ & $0,361(1)$ & $0,345(1)$ \\
\hline Área relativa $(\%)$ & 12 & 51 & 37 \\
\hline
\end{tabular}

$\mathrm{D}_{\mathrm{n}}=$ dubleto quadrupolar referente ao n-ésimo sítio cristalográfico (ajuste espectral)

$\mathrm{DQ}=$ desdobramento quadrupolar

$\delta=$ deslocamento isomérico

Os parâmetros verificados (Tab. 2) sugerem a presença de argilominerais e filossilicatos (Coey 1975, Hawthorne 1988, Murad 1998). Entretanto, não é possível distinguir de maneira inequívoca tais compostos, uma vez que, nesta temperatura, os parâmetros hiperfinos destes frequentemente se superpõem. Entretanto, segundo Goodman \& Bain (1978), há uma relação direta entre o número de coordenação e o deslocamento isomérico $(\delta)$ na clorita. Os valores obtidos usualmente são próximos e não permitem diferenciar se o ferro está em arranjo cis ou trans, o que nos leva a considerar uma regra semiempírica, que estabelece que uma coordenação trans conduza a um valor menor do desdobramento quadrupolar (De Grave et al. 1987).

Assim, os dados comparados com os da literatura indicam que o dubleto $\mathrm{D}_{1}$ corresponde ao $\mathrm{Fe}^{2+}$, em coordenação octaédrica, em arranjo trans (Goodman \& Bain 1978, De Grave et al. 1987, Parseval et al. 1991, Zazzi et al. 2006). Os dubletos $\mathrm{D}_{2}$ e $\mathrm{D}_{3}$ correspondem ao $\mathrm{Fe}^{3+}$ (De Grave et al. 1987), e podemos sugerir, com base nos baixos valores de $\delta(0,361$ e $0,345 \mathrm{~mm} / \mathrm{s})$, que estão em um arranjo trans na camada brucítica. Apesar de Zazzi et al. (2006) não adotarem o mesmo procedimento para cloritas naturais e sintéticas, devido aos valores do $\delta$ encontrados serem superiores a $0,41 \mathrm{~mm} / \mathrm{s}$ (Joswig et al. 1980), Phillips et al. (1980) distribuíram todos os elementos pesados $\left(\mathrm{Fe}^{3+}, \mathrm{Cr}^{3+} \mathrm{e} \mathrm{Ni}^{2+}\right)$ em sítios octaédricos $M 4$ da camada brucítica, que correspondem às distâncias entre as ligações na posição $M 4$.

Em termos de área relativa, os dados por EM indicaram que a clorita apresenta $12 \%$ de $\mathrm{Fe}^{2+}$ e $88 \%$ de $\mathrm{Fe}^{3+}$. Porém, esses dados foram obtidos a partir de uma amostra total de minerais, enquanto o conjunto de dados químicos obtidos por MEV-EDS consiste em análises pontuais em cristais únicos, nos quais, visualmente, a evolução da cloritização foi possível de ser observada ao microscópio óptico. Assim, os dados de Mössbauer não nos permitiram relacionar essa mesma proporção de $\mathrm{Fe}^{2+} \mathrm{e} \mathrm{Fe}^{3+}$ com as obtidas nas análises.

O conjunto das análises químicas (Tab. 1) em diversos cristais permitiu recalcular a fórmula estrutural para a clorita, com base em 28 oxigênios. São apresentadas as fórmulas de cloritas com diferentes composições. Todas as cloritas analisadas têm $\mathrm{Zn}$ na estrutura. - Sem Ti na fórmula:

$$
\begin{aligned}
& \left(\mathrm{Zn}^{2+}{ }_{2,92-3,33} \mathrm{Ca}_{0-0,07} \mathrm{Mg}_{5,44-5,62} \mathrm{Al}_{1,87-1,95} \mathrm{Fe}_{0,86-1,16}\right)_{12}[\mathrm{Si} \\
& \left.{ }_{5,35-6,18} \mathrm{Al}_{1,82-2,15}\right]_{8} \mathrm{O}_{20}(\mathrm{OH})_{16}
\end{aligned}
$$

- Sem Ti no tetraedro:

$$
\begin{aligned}
& \left(\mathrm{Zn}^{2+}{ }_{1,18-3,32} \mathrm{Ca}_{0-0,07} \mathrm{Mg}_{4,53-5,99} \mathrm{~K}_{0-1,31} \mathrm{Ti}^{4+}{ }_{0-0,16} \mathrm{Al}_{0-1,87} \mathrm{Fe}\right. \\
& \left.{ }_{1,45-4,2,2}\right)_{12}\left[\mathrm{Si}_{5,4-6,59} \mathrm{Al}_{2,57-3,28}\right]_{8} \mathrm{O}_{20}(\mathrm{OH})_{16}
\end{aligned}
$$

- Com Ti no tetraedro:

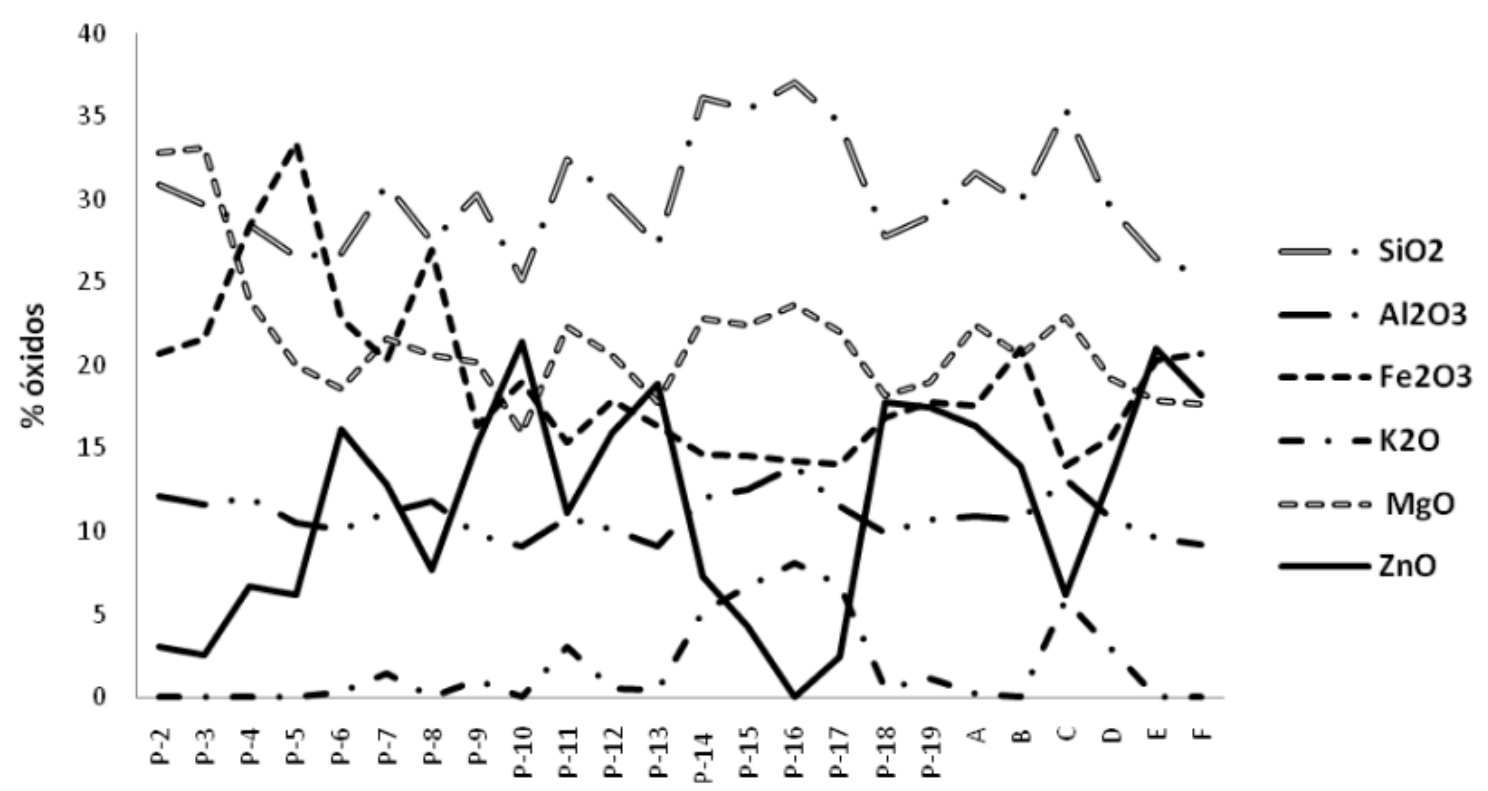

Figura 4 - Gráfico com os teores em $\mathrm{SiO}_{2}, \mathrm{Al}_{2} \mathrm{O}_{3}, \mathrm{Fe}_{2} \mathrm{O}_{3}, \mathrm{~K}_{2} \mathrm{O}, \mathrm{MgO}$ e $\mathrm{ZnO}$ (\% óxidos no eixo vertical) para os pontos analisados por EDS ( $n^{\circ}$ do ponto eixo horizontal). 


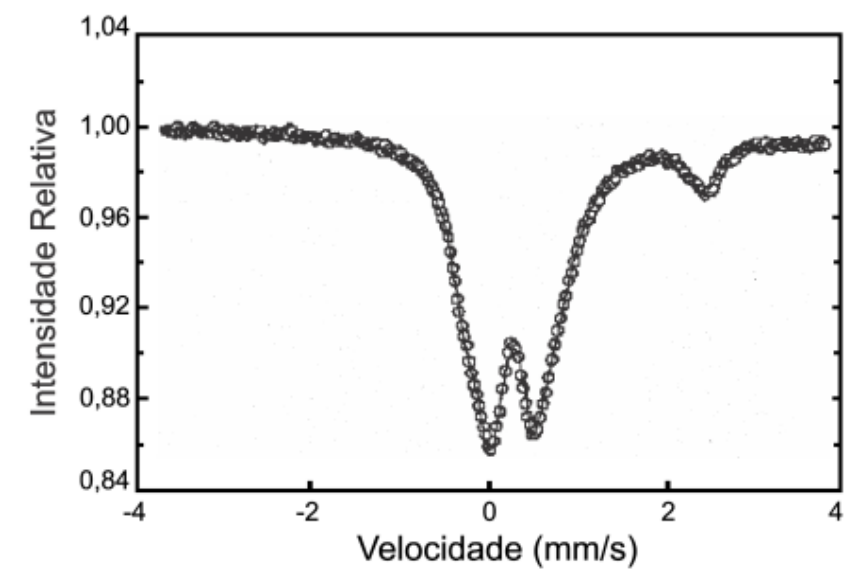

Figura 5 - Espectro Mössbauer a $T=77 \mathrm{~K}$ para a clorita de Canoas 1.

$$
\begin{aligned}
& \left(\mathrm{Zn}^{2+}{ }_{2,73-3,44} \mathrm{Mg}_{4,59-4,83} \mathrm{~K}_{0-0,05} \mathrm{Ti}^{4+}{ }_{0-0,07} \mathrm{Fe}_{1,45-4,2}\right)_{12}\left[\mathrm{Si}_{5,11-}\right. \\
& \left.{ }_{5,4} \mathrm{Al}_{2,58-2,77} \mathrm{Ti}^{4+}{ }_{0,01-0,18}\right]_{8} \mathrm{O}_{20}(\mathrm{OH})_{16}
\end{aligned}
$$

- Sem Ti no tetraedro:

$$
\begin{aligned}
& \left(\mathrm{Zn}^{2+}{ }_{068-3,76} \mathrm{Ca}_{0-0,03} \mathrm{Mg}_{5,12-6,75} \mathrm{~K}_{0-0,14} \mathrm{Ti}^{4+}{ }_{0-0,16} \mathrm{Al}_{0,11-0,14}\right. \\
& \left.\mathrm{Fe}_{2,64-4,17}\right)_{12}\left[\mathrm{Si}_{5,37-5,84} \mathrm{Al}{ }_{2,16-2,63}\right]_{8} \mathrm{O}_{20}(\mathrm{OH})_{16}
\end{aligned}
$$

- Com Ti no tetraedro:

$$
\begin{aligned}
& \left(\mathrm{Zn}^{2+}{ }_{068-3,76} \mathrm{Ca}_{0-0,03} \mathrm{Mg}_{5,12-6,75} \mathrm{Ti}^{4+}{ }_{0-0,11} \mathrm{Fe}_{2,64-4,17}\right)_{12}[\mathrm{Si} \\
& \left.{ }_{5,31-5,45} \mathrm{Al}_{2,21-2,53} \mathrm{Ti}^{4^{+}+}{ }_{0,02-0,09}\right]_{8} \mathrm{O}_{20}(\mathrm{OH})_{16}
\end{aligned}
$$

A conversão de óxidos para elementos foi realizada com o software Minfile e, utilizando o programa Excel, foram realizadas correlações estatísticas entre os elementos químicos nos diferentes sítios cristalográficos. Assim, correlações positivas foram indicadas entre $\mathrm{Si}-\mathrm{Mg}$ e Al-Ca, e correlações negativas entre Si-Zn,
$\mathrm{Si}-\mathrm{Fe}$, e Mg-Zn. Cabe ressaltar que, embora o Fe apresente correlação negativa com o $\mathrm{Zn}$, estes elementos apresentam em comum correlação negativa com o Si. Esse comportamento pode sugerir que houve entrada de $\mathrm{Fe}$, como $\mathrm{Fe}^{3+}$, e de $\mathrm{Zn}^{2+}$ nos tetraedros da estrutura cristalina da clorita. Porém, a correlação negativa entre o $\mathrm{Mg}$ e $\left(\mathrm{Zn}^{2+}+\mathrm{Fe}^{3+}\right)$ e a observação dos dados obtidos por EM, de que todo o ferro presente na clorita está em coordenação octaédrica, não confirmam esse comportamento. De fato, a presença de $\mathrm{Fe}^{3+}$ em coordenação tetraédrica em cloritas é raramente descrita na literatura, citando-se ocorrências de alguns filossilicatos (Dyar 1990) e clorita associada a depósitos de talco-clorita, nos Pirineus, França, esta última, provável herança da flogopita original (Parseval et al. 1991).

Os espectros de IV obtidos são típicos de clorita (Fig. 6), com bandas de absorção que podem estar afetadas pela mineralogia parasita presente no concentrado obtido de cloritas (smithsonita, cerussita, piromorfita, barita e anfibólio). A clorita apresenta dois modos de vibração característicos em espectros IV, uma banda de absorção em aproximadamente $3560 \mathrm{~cm}^{-1}$, denominada banda 2, e outra em aproximadamente $3420 \mathrm{~cm}^{-1}$, denominada banda 3 (Gadsden 1975, Shirozu 1985). Essas vibrações são atribuídas aos grupos $\mathrm{OH}$ situados nas interfaces formadoras da estrutura cristalina.

A presença de uma banda de absorção fraca na região de 3680-3620 $\mathrm{cm}^{-1}$, região designada de banda 1 , nas amostras de Canoas condizem com a encontrada em cristais de clorita com geometria trioctaédrica (Shirozu 1985).

As bandas de absorção em torno de $3500 \mathrm{~cm}^{-1}$ são complexas e Hayashi \& Oinuma (1967) e Shirozu (1980) definiram duas bandas principais, que dependem essencialmente das substituições tetraédricas da camada brucítica, acompanhadas de uma banda em

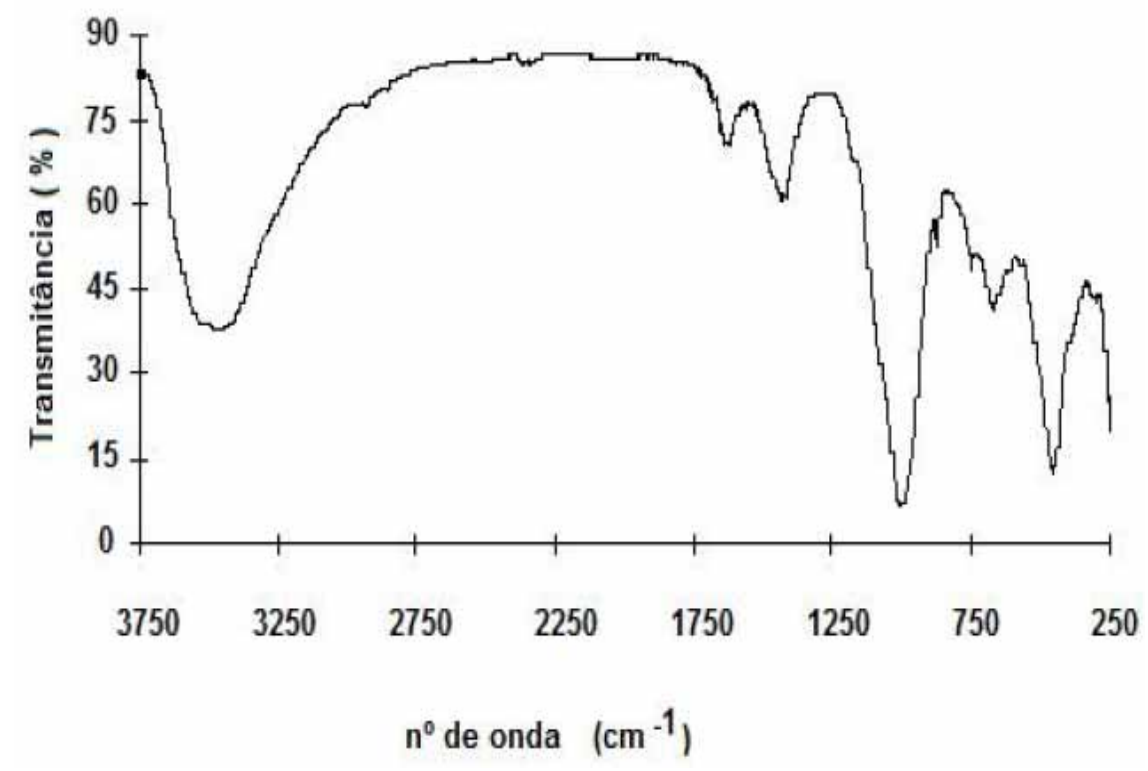

Figura 6 - Espectro IV para o conjunto de 8 amostras de clorita de Canoas 1. 
torno de $3650 \mathrm{~cm}^{-1}$ associadas à $\mathrm{OH}$ da camada 2:1. Nas amostras de Canoas esta banda em $3500 \mathrm{~cm}^{-1}$ é pouco visível, mas foi possível obtê-la a partir dos valores estatísticos calculados das 8 amostras analisadas. Assim, as três bandas definidas em 3565, 3503 e $3410 \mathrm{~cm}^{-1}$ devem corresponder à $\mathrm{OH}$ na camada brucítica associada ao oxigênio basal nas camadas $2: 1$ adjacentes. Uma possível interpretação é a de que essas bandas são causadas pelas diferenças entre as distâncias $\mathrm{OH} . . . \mathrm{O}$.

É conhecido que um aumento no teor de $\mathrm{Al}^{\mathrm{IV}}$ na estrutura cristalina leva a uma diminuição da frequência da banda 3 e a um concomitante aumento de sua intensidade relativa (Shirozu 1985). Na amostra de Canoas, esta banda de absorção ocorre em $3503 \mathrm{~cm}^{-1}$ (menor frequência) e é mais intensa, podendo ser atribuída a estiramentos $v \mathrm{OH}-\left(\mathrm{Fe}^{2+} / \mathrm{Fe}^{3+}\right)$ em simetria octaédrica e ao aumento de $\mathrm{Fe}_{\text {oct }}$ no mineral, ou também devido à presença de $\mathrm{Zn}$ na estrutura da clorita.

A banda da $\mathrm{OH}$ molecular em $1630 \mathrm{~cm}^{-1}$, típica em cloritas de qualquer composição, é notadamente bem correlacionada com os teores de $\mathrm{H}_{2} \mathrm{O}^{+}$. Uma banda em $1420 \mathrm{~cm}^{-1}$ significa a presença de carbonato em mistura com a amostra.

A banda dupla $1008 \mathrm{~cm}^{-1}$ e $996 \mathrm{~cm}^{-1}$ corresponde às ligações $\mathrm{Si}-\mathrm{O}-\mathrm{Si}$, e são típicas para clinocloro em 985-958 $\mathrm{cm}^{-1}$ (Shirozu 1980). Percebe-se uma correlação positiva entre o teor de Si no tetraedro e os valores obtidos para essa banda (Fig. 7), o que sugere que essa correlação seja função das substituições do Si pelo Al no tetraedro. De fato, as bandas se tornariam mais largas quanto maior o teor de $\mathrm{Al}^{3+}$, enquanto seriam menores nas espécies com maiores conteúdos de $\left(\mathrm{Fe}^{2+}+\mathrm{Fe}^{3+}\right.$ $+\mathrm{Mn}^{2+}$ ) e $\mathrm{Mg}^{2+}$ (Hayashi \& Oinuma 1967).

A banda de $750 \mathrm{~cm}^{-1}$ é quase ausente, e corresponde, provavelmente, aos carbonatos que também apresentam uma banda nesta região. A banda em $650 \mathrm{~cm}^{-1}$ varia em função do conteúdo no octaedro, principalmente de Fe. Em Canoas essa banda ocorre a $668 \mathrm{~cm}^{-1}$ e deve corresponder ao conteúdo de $\mathrm{Zn}$, que afeta diretamente sua intensidade.

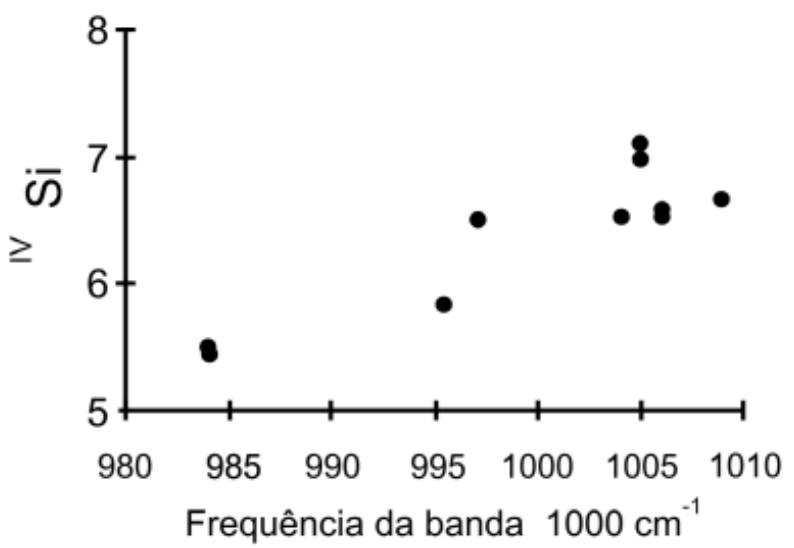

Figura 7 - Relação entre a posição da banda $1000 \mathrm{~cm}^{-1}$ e os conteúdos de Si no sítio tetraédrico na clorita.
A banda $450 \mathrm{~cm}^{-1}$ também depende do conteúdo no octaedro e é intensa e dissimétrica (em clinocloro a banda dupla está em $442-458 \mathrm{~cm}^{-1}$ ). A forma dissimétrica dessa banda é uma dissimetria complementar do coeficiente de variação, que se acredita seja o conteúdo octaédrico evolutivo do processo de cloritização segundo os teores dos elementos pesados, como o Fe e o $\mathrm{Zn}$ (Shirozu 1985).

As figuras $8 \mathrm{a}$ e $8 \mathrm{~b}$ apresentam correlações entre os teores de $\mathrm{Zn}$, recalculados para uma estrutura com 28 oxigênios, em função da intensidade das bandas de vibração $650 \mathrm{~cm}^{-1}$ e $450 \mathrm{~cm}^{-1}$, respectivamente.

CONCLUSÕES Os novos dados obtidos para as amostras de clorita, em conjunto com os trabalhos anteriormente realizados, permitiram indicar alguns pontos sobre o processo de cloritização que ocorre em flogopitas associadas ao minério de $\mathrm{Pb}-\mathrm{Zn}-\mathrm{Ag}$ de Canoas.

A clorita zincífera de Canoas é produto da alteração intempérica da flogopita e incorpora $\mathrm{Zn}$ na estrutura devido à alteração concomitante da esfalerita (Blot et al. 1995).

As análises por Mössbauer permitiram quantificar o $\mathrm{Fe}^{2+}(12 \%)$ e o $\mathrm{Fe}^{3+}(88 \%)$ e alocar estes íons na estrutura cristalina da clorita. Com base nestas análises e nos dados de correlação estatística, foi possível verificar que o $\mathrm{Fe}^{2+} \mathrm{e}$ o $\mathrm{Fe}^{3+}$ estão no sítio octaédrico em arranjo trans, e não existe Fe na camada tetraédrica da clorita.

Uma matriz de coeficientes de correlação entre as medidas efetuadas por IV na clorita de Canoas e os elementos estruturais, calculados a partir das análises químicas ao MEV/EDS, possibilitou verificar que os elementos que ocupam o sítio tetraédrico $\left(\mathrm{Si}, \mathrm{Al}^{\mathrm{IV}}\right)$ afetam a frequência das bandas 3500 e $1000 \mathrm{~cm}^{-1}$, assim como a intensidade da banda $750 \mathrm{~cm}^{-1}$. Dos elementos octaédricos ( $\mathrm{Al}^{\mathrm{IV}}, \mathrm{Fe}, \mathrm{Mg}$ e $\left.\mathrm{Zn}\right)$, sem distinção de localização, o $\mathrm{Al}^{\mathrm{IV}}$, $\mathrm{Fe}$ e $\mathrm{Zn}$, afetaram tanto a frequência das bandas $3500 \mathrm{~cm}^{-1}$ e $1000 \mathrm{~cm}^{-1}$ quanto das bandas $750 \mathrm{~cm}^{-1}, 650 \mathrm{~cm}^{-1} \mathrm{e} 450 \mathrm{~cm}^{-1}$. A incorporação do Zn na camada brucítica parece ser evidenciada pela banda $3500 \mathrm{~cm}^{-1}$, assim como afeta a intensidade das bandas $650 \mathrm{~cm}^{-1} \mathrm{e} 450 \mathrm{~cm}^{-1}$. A espectroscopia IV não nos dá elementos que permitam indicar a presença de Zn na camada tetraédrica.

Tuddenham \& Lyon (1958 apud Hayashi \& Oinuma 1967) descrevem que a máxima absorção da $\mathrm{OH}$ em Fe-cloritas seria entre $3560-3400 \mathrm{~cm}^{-1}$, que são valores inferiores aos observados para Mg-cloritas cujos valores estão entre $3580-3417 \mathrm{~cm}^{-1}$. Em geral, com o aumento da distância $\mathrm{OH}$...O, a frequência de vibração aumenta e, assim, a distância entre a OH da camada brucítica e o oxigênio tetraédrico na Fe-clorita seria menor do que o da Mg-clorita. Com base nos espectros de IV, a clorita de Canoas seria uma Fe-clorita, porém, a análise da composição química indica, em porcentagem de elemento, ser uma clorita com composição mais rica em Mg. No entanto, os mesmos autores sugerem que as bandas podem ser influenciadas por diferentes cátions na camada brucítica, e este comportamento poderia ser 


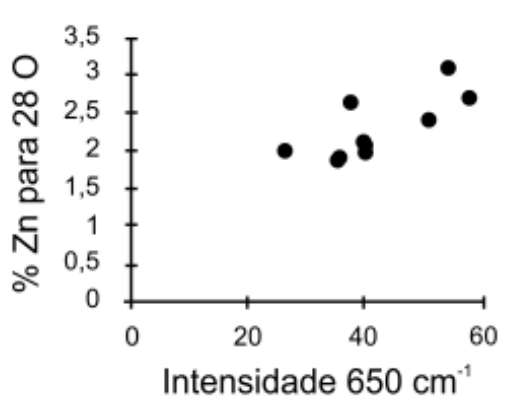

a

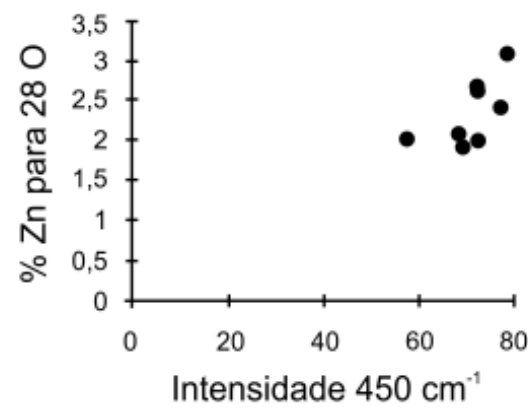

b

Figura 8 - a) Correlação entre o conteúdo octaédrico em Zn e a intensidade da banda de vibração $650 \mathrm{~cm}^{-1}$ na clorita; (b) Correlação entre os conteúdos octaédricos em Zn e a intensidade da banda de vibração $450 \mathrm{~cm}^{-1}$.

decorrente da presença de $\mathrm{Zn}$, o que explicaria a presença da banda em $3503 \mathrm{~cm}^{-1}$.

A interpretação conjunta dos dados obtidos pelas diferentes análises possibilitou verificar que com o aumento do grau de intemperização existe uma troca gradual na composição da flogopita, com decréscimo de $\mathrm{MgO}, \mathrm{SiO}_{2}$ e $\mathrm{K}_{2} \mathrm{O}$ e aumento relativo de $\mathrm{FeO}$ e $\mathrm{ZnO}$. A incorporação de $\mathrm{Zn}$ está associada ao processo de oxidação do minério, existindo um trend na composição da clorita que modifica as relações entre o $\mathrm{Si}^{\mathrm{IV}}$ e o $\mathrm{Al}^{\mathrm{IV}}$; o $\mathrm{Al}^{\mathrm{VI}}$ e o $\mathrm{Fe}^{\mathrm{VI}}$ e, em menor extensão, o par Mg-Zn.

Assim, os novos dados obtidos permitem indicarmos que o processo de cloritização das flogopitas em Canoas 1 ocorre com a incorporação de $\mathrm{Zn}$, na camada octaédrica, em substituição ao Si e Mg na estrutura.

\section{Referências}

Bailey S.W. 1988. Structures of layer silicates. In: Brindle G.W. and Brown G. (eds.) Crystal Structures of Clay Minerals and their X-ray Identification. Londres, Mineralogical Society, p. 1-124.

Bettencourt J.S., Daitx E.C., Moreira M.Z., Matsui E. 1992. Estudo isotópico pelos métodos ${ }^{13} \mathrm{C}^{12} \mathrm{C},{ }^{18} \mathrm{O} /{ }^{160}, d D e$ ${ }^{34} S^{\beta 2} S$ dos depósitos de chumbo-zinco-prata do vale do Ribeira, Estados de São Paulo e Paraná. São Paulo, FAPESP. (relatório de Pesquisa Processo 90/2139-2).

Blot A., Imbernon R.A.L. 2000. Caractérization par analyse thermique de la constitution cristallochimique de diverses chlorites zinciféres. C. R. Acad. Sci. Paris, Sciences de la Terre e des Planètes: 330(7):469-472.

Blot A., Oliveira S.M.B., Magat P. 1995. La chloritisation supergène zincifère des phlogopites de Canoas (PR, Brésil). C. R. Acad. Sci. Paris, Sciences de la Terre e des Planètes, 321(II a):651-658.

Brindley G.W., Brown G. (eds.) 1984. Crystal structures of Clay Minerals and their X-ray identification. Londres, Min. Soc., 504 p.

Coey J.M.D. 1975. Clay minerals and their transformations studied with nuclear techniques: the contribution of Mössbauer spectroscopy: At. Energy Rev. 18:1-124.

Daitx E.C. 1996. Origem e evolução dos depósitos sulfetados tipo-Perau (Pb-Zn-Ag), com base nas jazidas Canoas e Perau (Vale do Ribeira, PR). Tese de Doutorado, Instituto de Geociências e Ciências Exatas, Universidade Estadual Paulista Júlio de Mesquita Filho, Rio Claro, 453 p.
Dyar M.D. 1990. Mössbauer spectra of biotite from metapelites. American Mineralogist, 75:656-666.

De Grave E., Vandenbruwaene J., Van Bockstael M. 1987. ${ }^{57} \mathrm{Fe}$ Mössbauer spectroscopic analysis of chlorite. Phys. Chem. Minerals, 15:173-180.

Dunn P.J., Peacor D.R., Ramik R.A., Su S.C., Rouse R.C. 1987. Franklinfurnaceite, a $\mathrm{Ca}-\mathrm{Fe}^{3+}-\mathrm{Mn}^{3+}-\mathrm{Mn}^{2+}$ a zincosilicate isotopic with chlorite, from Franklin, New Jersey. American Mineralogist, 72:812-815.

Hawthorne F.C. 1988. Mössbauer spectroscopy. Reviews in Mineralogy, 18:255-340.

Fleischer R. 1976. A pesquisa de chumbo no Brasil. In: SBG, Congresso Brasileiro de Geologia, 29, Anais, p. 19-32.

Foster M.D. 1962. Interpretation of the composition and a classification of the chlorites. U.S. Geol. Surv. Prof. Paper, 414-A:1-33.

Franco D.R., Berquó T.S., Imbernon R.A.L., Partiti C.S.M., Enzweiler J. 2007. Environmental monitoring of magnetic iron phases of urban water reservoir lake sediments (Taiaçupeba Lake, metropolitan region of São Paulo, Brazil) by using Mössbauer spectroscopy. Environmental Geology, 52:831-842.

Gadsden J.A. 1975. Infrared spectra of minerals and related inorganic compounds. London, Butterworth \& Co., $277 \mathrm{p}$.

Goodman B.A., Bain D.C. 1978. Mössbauer spectra of chlorites and their decomposition products. In: Elsevier Scientific Pub. Co. Amsterdan, International Clay Conference, 6, Anais, p. 65-74. 
Caracterização de clorita portadora de Zn por espectroscopia Mössbauer (EM) e espectroscopia infravermelho (IV) - uma ocorrência associada ao depósito de Pb-Zn-Ag de Canoas, PR, Brasil

Hayashi H., Oinuma K. 1967 Si-O absorption band near $1000 \mathrm{~cm}^{-1}$ and $\mathrm{OH}$ absorption bands of chlorite. American Mineralogist, 52:1206-1210.

Imbernon R.A.L. 1998. Os chapéus de ferro associados aos depóstios de Canoas (Pb-Zn-Ag), Adrianópolis (PR) e $O$ 'Toole (Cu-Ni-EGP), Fortaleza de Minas (MG). Tese de Doutorado, Instituto de Geociências, Universidade de São Paulo, São Paulo, 120 p.

Imbernon R.A.L., Blot. A., Oliveira S.M.B., Magat P. 1999. Os chapéus de ferro associados ao depósito de $\mathrm{Pb}-\mathrm{Zn}$ Ag na região de Canoas, Adrianópolis, PR - Evolução geoquímica e mineralógica. Geochim. Brasil., 13(2):145-161.

Joswig W., Fuess H., Rothbauer R., Takéuchi Y., Mason S.A. 1980. A neutron diffraction study of a one-layer triclinic chlorite (pennite). American Mineralogist, 65:349-352.

Murad E. 1998. Clays and clay minerals: what can Mössbauer spectroscopy do to help understand them? Hyperfine Interaction, 117:39-70.

Newman A.C.D., Brown G. 1987. The chemical constitution of clay. In: Newman, A.C.D. (ed.) Chemistry of Clays and Clays Minerals. Essex, Longman Scientific and Technical, p. 1-128.

Parseval P., Fournes L., Fortune J.P., Moine B., Ferret J. 1991. Distribution du fer dans les chlorites par spectrométrie Mössbauer $\left({ }^{57} \mathrm{Fe}\right): \mathrm{Fe}^{3+}$ dans les chlorites du gisement de talc-chlorite de Trimouns (Pyrénées, France). C. R. Acad. Sci. Paris, Sciences de la Terre e des Planètes, 312(2):1321-1326.

Phillips T.L., Loveless J.K., Bailey S.W. 1980. $\mathrm{Cr}^{3+}$ coordination in chlorites: a structural study of ten chromian chlorites. American Mineralogist, 54:112-122.

Rule A., Radke F. 1988. Baileychlore, the Zn end member of the trioctahedral chlorite series. American Mineralogist, 73:135-139.

Shirozu H. 1980. Cation distribution, sheet thickness, and $\mathrm{O}-\mathrm{OH}$ space in trioctahedral chlorites-an X-ray an infrared study. Miner. Journal, 10:14-34.

Shirozu H. 1985. Infrared spectra of trioctahedral chlorites in relation to chemical composition. Clay Sci. 6:167-176.

Tuddenham W.M., Lyon R.J.P. 1958. Relation of infra-red spectra and chemical analysis for some chlorite and related minerals. Anal.Chem. 31:377-380.

Zazzi Å., Hirsch T.K., Leonova E., Kaikkonen A., Grins J., Annersten H., Edén M. 2006. Structural investigations of natural and synthetic chlorite minerals by X-ray diffraction, Mössbauer spectroscopy and solid-state nuclear magnetic resonance. Clays and Clay Minerals, 54(2):252-265.

Manuscrito ID 15686

Submetido em 03 de dezembro de 2010 Aceito em 04 de outubro de 2011 